\title{
Two-color quantitative multiplex methylation-specific PCR
}

\author{
Theresa Swift-Scanlan ${ }^{1,2}$, Amanda Blackford ${ }^{1}$, Pedram Argani ${ }^{1}$, Saraswati Sukumar ${ }^{1,2}$, and Mary Jo Fackler ${ }^{1}$ \\ BioTechniques 40:210-219 (February 2006) \\ doi $10.2144 / 000112097$
}

\begin{abstract}
In recent years, several methylation-specific PCR-based techniques have been developed to identify and characterize hypermethylation of $C p G$ dinucleotides with the primary goal of elucidating a better understanding of the role of DNA methylation in important biological processes, such as chromosome X inactivation and carcinogenesis. The specificity of methylation-specific PCR (MSP) techniques relies on amplifying sodium bisulfite-treated DNA with primers specific to predicted sequences of unmethylated and methylated DNA within the gene of interest. In the past, unmethylated and methylated reactions were singleplex and performed in separate wells. In this paper, we report a modification of the real-time quantitative multiplex MSP (QM-MSP) technique of Fackler and colleagues that can be applied to any real-time MSP experiment. Although co-amplification with multiple fluorophores is common in standard reverse transcription PCR (RT-PCR), MSP presents unique challenges both mechanistically and operationally that must be overcome in order to successfully co-amplify two methylation-specific targets. In this two-color modification, unmethylated and methylated primer/probe sets are successfully co-amplified in the same reaction using FAM ${ }^{\mathrm{TM}}$ - and VIC ${ }^{\circledR}$-labeled probes. Our modification decreases the cost and time of each real-time experiment by allowing increased throughput of clinical samples and by doubling either the number of genes or the number of samples that can be analyzed per real-time plate.
\end{abstract}

\section{INTRODUCTION}

Hypermethylation of $\mathrm{CpG}$ islands in promoter regions of numerous genes involved in cell growth, differentiation, and DNA repair processes is now recognized as an important and early event in carcinogenesis (1-7). Methylation of the cytosine residue of $\mathrm{CpG}$ dinucleotides epigenetically silences gene expression through two main mechanisms: ( $i$ ) by triggering a series of events leading to a closed chromatin structure that inhibits transcription and (ii) by altering the DNA binding and specific action of transcriptional regulatory proteins via methylation of specific cytosines located within their binding sites $(8,9)$.

Given the importance of cytosine methylation in several key biological processes, such as genomic imprinting, chromosome $\mathrm{X}$ inactivation, and carcinogenesis, there has been great interest in developing methods to detect and quantify methylation. One major breakthrough central to the majority of these methylation detection methods has been the use of sodium bisulfite $(\mathrm{NaBi})$ to treat genomic DNA, a process that converts all nonmethylated cytosines to uracil (later replicated as thymidine during PCR), while leaving methylated cytosines intact $(10,11)$. The ability to predict sequences of methylated, $\mathrm{M}$, or unmethylated, U, DNA after $\mathrm{NaBi}$ conversion facilitated the subsequent development of a PCR-based method called methylation-specific PCR (MSP) (12). In MSP, the specificity of methylation detection hinges on primer design, whereby two sets of primers (an M and a $\mathrm{U}$ primer set) are used to characterize methylation for each gene.

Because standard MSP is a qualitative reaction that renders either positive or negative results, it is not informative regarding relative amounts of methylation; a critical problem that is magnified by the presence of low levels of DNA methylation in some normal tissues (13-15). To address this concern, a number of quantitative MSP techniques have been developed in recent years to evaluate either relative amounts of DNA promoter hypermeth- ylation between normal and affected tissues or methylation of a specific $\mathrm{CpG}$ dinucleotide. These include realtime applications such as quantitative MSP (also known as MethyLight) (16), HeavyMethyl (17), MethylQuant (18), and quantitative multiplex MSP (QM-MSP) (15). To our knowledge, all of these methods currently use two reaction wells per sample to characterize methylation of a single gene. Specifically, in MethyLight, the M reaction is carried out in one well, while a reference gene such as actin is amplified in the second well (16). Similarly, the gel-based MSP methods of Herman et al. (12) and the real-time QM-MSP method of Fackler et al. (15) use one well for primers specific to the unmethylated $U$ gene fraction and a second well for primers specific to the methylated $\mathrm{M}$ gene fraction.

MSP presents unique challenges that distinguish it from traditional PCR, including successful modification of genomic DNA template via $\mathrm{NaBi}$ treatment prior to MSP and the need to characterize both the methylated

\footnotetext{
${ }^{1}$ Johns Hopkins University School of Medicine and ${ }^{2}$ The Johns Hopkins University School of Nursing, Baltimore, MD, USA
} 
and unmethylated fractions of a particular target DNA; or in the case of some quantitative MSP techniques, to measure the methylated fraction of a gene relative to an unmethylated reference gene such as actin. Moreover, the use of multiple fluorophores in any real-time quantitative MSP presents additional concerns above and apart from traditional reverse transcription PCR (RT-PCR) that are related to quantification of the U+M DNA targets in real-time and the added competition between $\mathrm{U}+\mathrm{M}$ probes and primers for similar targets. Finally, the special requirements for $\mathrm{U}+\mathrm{M}$ real-time primers and probes with complementary melting temperatures $\left(\mathrm{T}_{\mathrm{m}} \mathrm{s}\right)$ are more difficult to achieve, because all unmethylated cytosines are converted to uracil during $\mathrm{NaBi}$ treatment. All of these factors contribute to the complexity of real-time MSP.

While several quantitative MSP techniques offer between 10- to 100fold increases in sensitivity over standard gel-based MSP, they are more costly, and the real-time reaction is further compromised by singleplexing of $\mathrm{U}$ and $\mathrm{M}$ primer/probe sets, which limits the number of samples that can be analyzed per plate. As quantitative MSP techniques hold promise for translation into the clinical setting, improved efficiency and affordability is needed for their application as diagnostic tests.

We now report an improvement in the real-time step of QM-MSP, which utilizes two primer/probe sets labeled with fluorophores of FAM ${ }^{\mathrm{TM}}$ and VIC ${ }^{\circledR}$, respectively, to co-amplify two methylation-specific DNA targets in the same well. In this paper, we compare the realtime step of the traditional one-color QM-MSP method with three possible protocols of our improved method: (A) a two-gene reaction wherein $\mathrm{U}_{1}+\mathrm{U}_{2}$ (unmethylated primer/probe sets for genes $1+2$ ) and $M_{1}+M_{2}$ (methylated primer/probe sets for genes $1+2$ ) are co-amplified in one well, respectively; (B) a two-gene reaction where $\mathrm{U}_{1}+\mathrm{M}_{2}$ (an unmethylated primer/probe set for gene 1 and a methylated primer/ probe set for gene 2) and $\mathrm{M}_{2}+\mathrm{U}_{1}$ (a methylated primer/probe set for gene 2 and an unmethylated primer/probe set for gene 1) are co-amplified in the same well; and (C) a single-gene reaction where the $U_{1}$ and $M_{1}$ primers and probes for the same gene are co-amplified in one well; hereafter referred to as protocols $\mathrm{A}, \mathrm{B}$, and $\mathrm{C}$, respectively. While co-amplification with two or more fluorophores has been performed for standard RT-PCR (1921), to our knowledge, this is the first report for real-time MSP applications that either two genes or U+M primer/ probe sets for the same gene have been simultaneously quantified in a single well. This two-color modification can be applied not only to QM-MSP, but to any real-time MSP experiment.

\section{MATERIALS AND METHODS}

\section{DNA Extraction}

Hematoxylin and eosin (H\&E)stained 5- $\mu \mathrm{m}$ sections of breast tumor tissue were deparaffinized in xylene, scraped into a collection tube, and treated with proteinase K (15). DNA was precipitated with ethanol according to standard protocol. For MDA-MB-231 cells (methylated control) and human sperm DNA (HSD; unmethylated control), DNA was purified either via phenol-chloroform extraction, or the Puregene $^{\circledR}$ DNA Purification kit (Gentra Systems, Minneapolis, MN, USA), respectively.

\section{Sodium Bisulfite Treatment}

DNA derived from breast tumor tissue, control breast tissue obtained from reduction mammoplasty of healthy women, HSD, or MDA-MB-231 cells were $\mathrm{NaBi}$-treated according to the standard protocol for MSP of Herman et al. (12). NaBi treatment converts nonmethylated cytosine residues to uracil (later replicated as thymidine during PCR), whereas methylated cytosines remain unchanged.

\section{Quantitative Multiplex Methylation- Specific PCR}

QM-MSP was performed in two sequential PCR steps as described by Fackler et al. (15).

In step 1, multiplex PCR was performed using external primers independent of DNA methylation status to co-amplify six genes (RASSF1A, RAR $\beta, T W I S T$, Cyclin D2, ER $\alpha$, and HIN1) using as little as $40 \mathrm{pg} \mathrm{NaBi}$ treated genomic DNA as the template in a $25-\mu \mathrm{L}$ PCR volume (15). The final PCR product was then diluted 1:5 or more in sterile distilled water, depending on the concentration of input DNA. In step 2, in the quantitative reaction, real-time MSP was performed on $1 \mu \mathrm{L}$ of the diluted PCR product from step 1 . The second step was carried out in a $25-\mu \mathrm{L}$ PCR final reaction volume with $1.25 \mathrm{U}$ Platinum DNA Taq polymerase (Invitrogen, Carlsbad, CA, USA), $1 \times$ ROX $^{\mathrm{TM}}$ passive reference dye (Invitrogen), $1 \times$ buffer [16.6 mM $\left(\mathrm{NH}_{4}\right)_{2} \mathrm{SO}_{4}, 67.0 \mathrm{mM}$ Tris, $\mathrm{pH} 8.8,6.7 \mathrm{mM} \mathrm{MgCl} 2,10.0 \mathrm{mM}$ $\beta$-mercaptoethanol, $0.1 \%$ dimethyl sulfoxide (DMSO)], $200 \mu \mathrm{M}$ dNTP, and $800 \mathrm{nM}$ of each primer set. The real-time assay was performed using an ABI PRISM ${ }^{\circledR}$ 7900HT Sequence Detector (Applied Biosystems, Foster City, CA, USA) as per protocol (15), with the exception that probe concentrations were optimized for each gene depending on the fluorescent reporter dye label. Optimal concentrations were determined experimentally from a $100 \mu \mathrm{M}$ probe stock to be $0.050 \mu \mathrm{L} /$ reaction for the FAM probe (Applied Biosystems) and $0.067 \mu \mathrm{L} /$ reaction for the VIC probe (Applied Biosystems), for a final concentration of $200 \mathrm{nM}$ and $268 \mathrm{nM}$, respectively. The standard curve, control, and sample DNAs were prepared essentially as described in Fackler et al. (15). Percent methylation for each gene was calculated as $[\mathrm{M} /(\mathrm{U}+\mathrm{M})] \times 100$ using the absolute quantification method as described (15).

\section{Real-Time Probes}

Real-time probes for each coamplified unmethylated and methylated gene/primer set were either labeled with a $5^{\prime}$-FAM/TAMRA ${ }^{\text {TM }}{ }_{-} 3^{\prime}$ or a $5^{\prime}$ VIC/TAMRA-3' reporter/quencher dye combination (Applied Biosystems). For example, for a two-gene reaction, we used the following $\mathrm{U}_{1}+\mathrm{U}_{2}$ and $\mathrm{M}_{1}+\mathrm{M}_{2}$ combinations: RASSF1AM-FAM + RAR $\beta$-M VIC in one well and RASSF1A-U VIC + RAR $\beta-U$ FAM in the second well for a given 
sample. Similarly, examples of $\mathrm{U}_{1}+\mathrm{M}_{1}$ same-gene two-color combinations were as follows: RASSF1A-M FAM + RASSF1A-U VIC in the same well and RAR $\beta-M$ VIC + RAR $\beta-U$ FAM in the same well.

\section{RESULTS}

In order to achieve significant reduction in costs and increase the throughput of clinical samples for methylation studies, we sought to develop a two-color modification of QM-MSP whereby two DNA targets are simultaneously co-amplified in real-time using TaqMan $^{\circledR}$ probes (Applied Biosystems) with hydrolysisbased chemistry. Figure 1 illustrates the two-step process of QM-MSP. In brief, QM-MSP uses two sequential PCRs. The first is a multiplex PCR, whereby a panel of genes is amplified from $\mathrm{NaBi}$ treated genomic DNA independent of the DNA methylation status for each gene (denoted by different colors in Figure 1). In the second quantitative real-time MSP step, PCR products of the first reaction are diluted and used as templates. Percent methylation is calculated by the absolute quantification method using a standard curve for each target DNA. In the traditional QM-MSP method, the copy number of $\mathrm{U}$ and $\mathrm{M}$ products is calculated separately in two wells (15). Our goal of performing co-amplification by combining genespecific primer/probe sets for either one- or two-gene methylation-specific DNA targets (denoted by light blue and red) is illustrated by the A, B, and $\mathrm{C}$ two-color protocols described in this paper.

Probe optimization is critical for each DNA target combination due to interactive effects and competition that can occur when co-amplifying with multiple fluorophores $(22,23)$. The added complexity and considerations of performing MSP over standard RT-PCR in the real-time environment, coupled with the limited number of reporter dyes available for successful multiplexing on the ABI platform, made finding a successful reporter dye combination for our real-time MSP application extremely difficult.

\section{1st Step: Multiplex PCR}

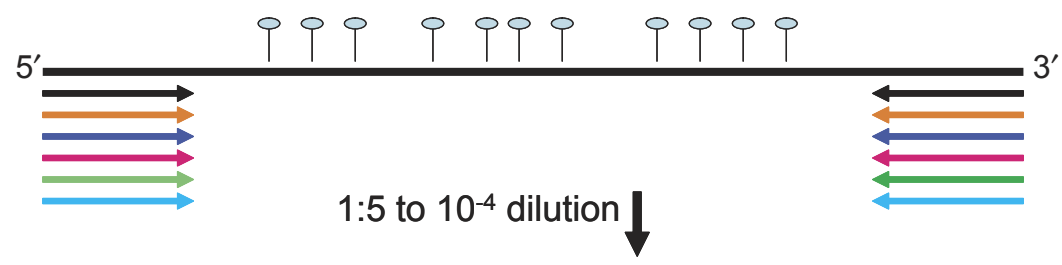

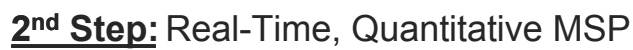

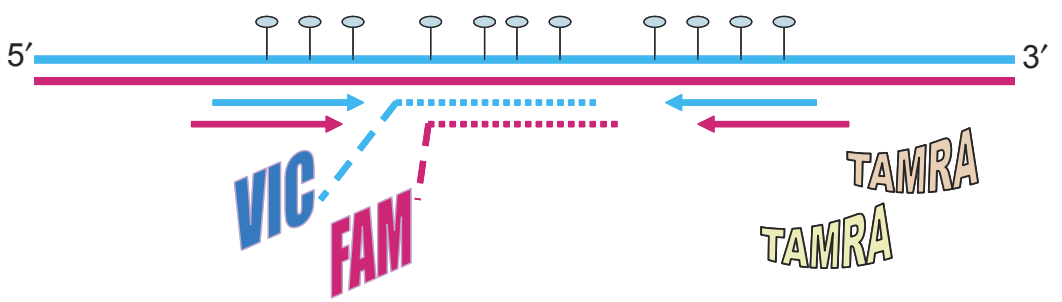

Symbol signifies a $\mathrm{CpG}$ dinucleotide

Figure 1. Two-color quantitative multiplex methylation-specific PCR (QM-MSP) showing two DNA targets co-amplified in the same real-time reaction well. The first step of QM-MSP is a multiplexed PCR of a panel of genes amplified from sodium bisulfite (NaBi)-treated genomic DNA with primers external to the $\mathrm{CpG}$ island and independent of DNA methylation status. Product sizes range from 150-325 bp. The second step shows two DNA targets (denoted by red and blue) co-amplified in real-time via the use of FAM- and VIC-5'-labeled probes using the diluted multiplexed PCR products obtained from the first step as a template. Permutations of the second step can include sets of oligonucleotide primers and probes to target DNAs of the same or different genes. MSP, methylation-specific PCR; VIC and FAM, 5'-labeled reporter dyes for the 27-bp probes covering on average 3 to 4 CpG dinucleotides per gene; and TAMRA, the $3^{\prime}$ real-time quencher dye.

Further, successful co-amplification is not a given even if each fluorophore in the combination performs well in singleplex. For example, we first selected a combination of FAM and Cal-Orange ( $\mathrm{CalO})$ labeled probes (Biosearch Technologies, Novato, CA, USA), because they meet the requirements of having sufficient intensity and nonoverlapping absorption spectra when amplified alone. However, this reaction was inefficient when the two probes were co-amplified due to interactive effects that caused the $\mathrm{CalO}$ signal intensity to fall at or below the cycle threshold $\left(\mathrm{C}_{\mathrm{T}}\right)$ (Figure 2 ). Inefficient co-amplification with $\mathrm{CalO}$ occurred in repeated experiments for either two genes or $\mathrm{U}_{1}+\mathrm{M}_{1}$ primer/ probe sets of the same gene. Figure 2A shows a standard QM-MSP curve using a RASSF1A-U CalO-labeled probe that performed well when amplified alone, but decreased by more than half its signal amplitude when co-amplified with the RAR $\beta-M$ FAM-labeled probe (Figure 2B). Co-amplification was inefficient regardless of whether TAMRA or Black Hole Quencher ${ }^{\circledR}{ }_{-1}$ (BHQ1; Biosearch Technologies) was used as the quencher dye for either probe (data not shown).

Next, the two-gene reactions were conducted using a combination of VIC- and FAM-labeled probes in the same well. This particular combination was chosen because these fluorophores have distinct absorption spectra and strong spectral intensities (see Applied Biosystems User Bulletin \#5, ABI PRISM 7700, 2001). In contrast to the results shown in Figure 2, A and B, Figure $2 \mathrm{C}$ shows a standard QM-MSP curve using a RASS-U VIC probe with a very robust amplification signal when performed alone and with a mildly diminished, but still strong, signal well above the $\mathrm{C}_{\mathrm{T}}$ when co-amplified with the RAR $\beta$-M FAM probe.

Even when an ideal probe/label combination such as FAM and VIC is found, interactive effects still occur during the real-time MSP that require further optimization. Figure 3 highlights these interactive effects and shows probe optimization for $\mathrm{M}_{1}+\mathrm{M}_{2}$ primers and probes, each set incorporating either labeled FAM or VIC fluorophores, respectively, for two 
genes known to be methylated in some breast cancers; namely RASSF $1 A$ and $R A R \beta$. Although the $\mathrm{M}_{1}+\mathrm{M}_{2}$ primers and probes for the two genes were co- amplified in the same well, Figure 3A shows only the data for the RAR $\beta-M$ DNA target reaction at three different concentrations $(0.05,0.067$, and 0.083
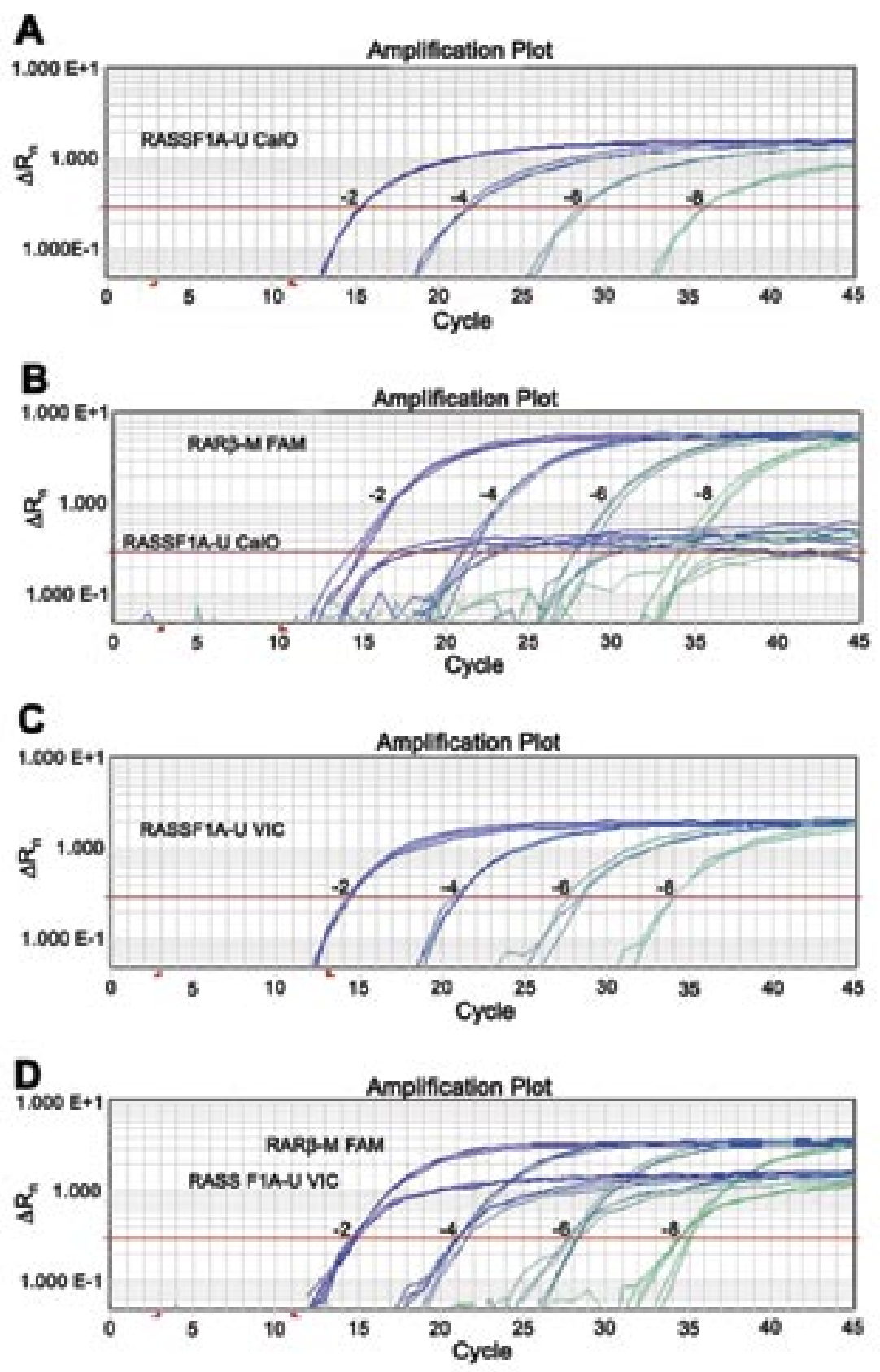

Figure 2. Comparative interactive effects of co-amplification during quantitative multiplex methylation-specific PCR (QM-MSP): CalO + FAM- versus VIC + FAM-labeled probes. Real-time plots showing signal intensity of RASSF1A-U CalO when amplified alone (A), as compared with a significantly decreased signal at or below the cycle threshold when co-amplified with a second gene, RAR $\beta$-M FAM (B). Conversely, panel C shows the signal intensity of RASSF1A-U VIC amplified alone, which unlike CalO, retains its strong signal when co-amplified with a second gene, RARB-M FAM (D). Template DNA consisted of a standard curve of equal amounts of human sperm DNA (HSD) and MDAMB-231 diluted to $10^{-2}, 10^{-4}, 10^{-6}$, and $10^{-8}$ in all four panels, respectively. Plotted is $\Delta \mathrm{R}_{\mathrm{n}}$ versus PCR cycle. y-axis: $\Delta \mathrm{R}_{\mathrm{n}}$, fluorescence emission signal of CalO, FAM, or VIC reporter dye/passive reference ROX dye across each real-time cycle; $x$-axis: cycle, cycle number of the real-time PCR. The horizontal line indicates the threshold; cycle threshold $\left(\mathrm{C}_{\mathrm{T}}\right)$, cycle number at which the $\mathrm{R}_{\mathrm{n}}$ exceeds the baseline by the value of the threshold.

$\mu \mathrm{L} /$ reaction of a $100 \mu \mathrm{M}$ stock; or 200, 268, and 332, respectively) of the RAR $\beta$-M-VIC probe. As expected, there is an increase in amplitude and a decrease in $\mathrm{C}_{\mathrm{T}}$ as the amount of RAR $\beta$ $\mathrm{M}-\mathrm{VIC}$ increases.

The interactive effects between the FAM- and VIC-labeled probes are apparent in Figure 3B, which shows that although the RASSF1A-M-FAM probe is held constant at $0.05 \mu \mathrm{L} /$ reaction, merely increasing the concentration of the co-amplified RAR $\beta$-MVIC probe partner, causes a decrease in the $\mathrm{C}_{\mathrm{T}}$ and an increase in amplitude of the RASSF1A-M-FAM reaction as the VIC probe concentration increases. We found optimal probe concentration for the genes in our analysis to be 0.05 and $0.067 \mu \mathrm{L} /$ reaction for the FAM and VIC probes (final concentration 200 and $268 \mathrm{nM}$ ), respectively. Thus, successful co-amplification of two methylation-specific target DNAs was accomplished with primer sets that utilize both FAM- and VIC-labeled probe sets in the same well.

Subsequently, we tested the application of the FAM- and VIC-labeled probe combination using three distinct protocols and compared the efficiencies of each with the established singleplex QM-MSP method. Table 1 compares percent methylation values between the one-color QM-MSP method and three permutations of our two-color modification of the real-time step of QM-MSP: (A) $\mathrm{U}_{1}+\mathrm{U}_{2}$ and $\mathrm{M}_{1}+\mathrm{M}_{2}$ twogene reaction; (B) $\mathrm{U}_{1}+\mathrm{M}_{2}$ and $\mathrm{M}_{1}+\mathrm{U}_{2}$ two-gene reaction; and (C) a $\mathrm{U}_{1}+\mathrm{M}_{1}$ reaction for the same gene. We have successfully co-amplified A, B, and C primer/probe sets specific for RASSF $1 A$ and RAR, CyclinD 2 and TWIST, and $E R \alpha$ and HIN1-six genes known to be methylated in breast cancer (Table 1, data for $E R \alpha$ and HIN1 not shown). Pearson correlation tests between percent methylation values obtained from one-color and two-color QMMSP for each combination were run separately for breast cancer and normal reduction mammoplasty tissue controls. Generalized estimating Equations (24) were used to test if the relationship between one-color and two-color QM-MSP values differed across A, B, and $\mathrm{C}$ approaches. An exchangeable correlation structure was specified, 
and percent methylation values were transformed as the natural $\log (\ln ) \times$
$(\% \mathrm{M}+1)$ to fulfill normality assumptions. Percent methylation values were
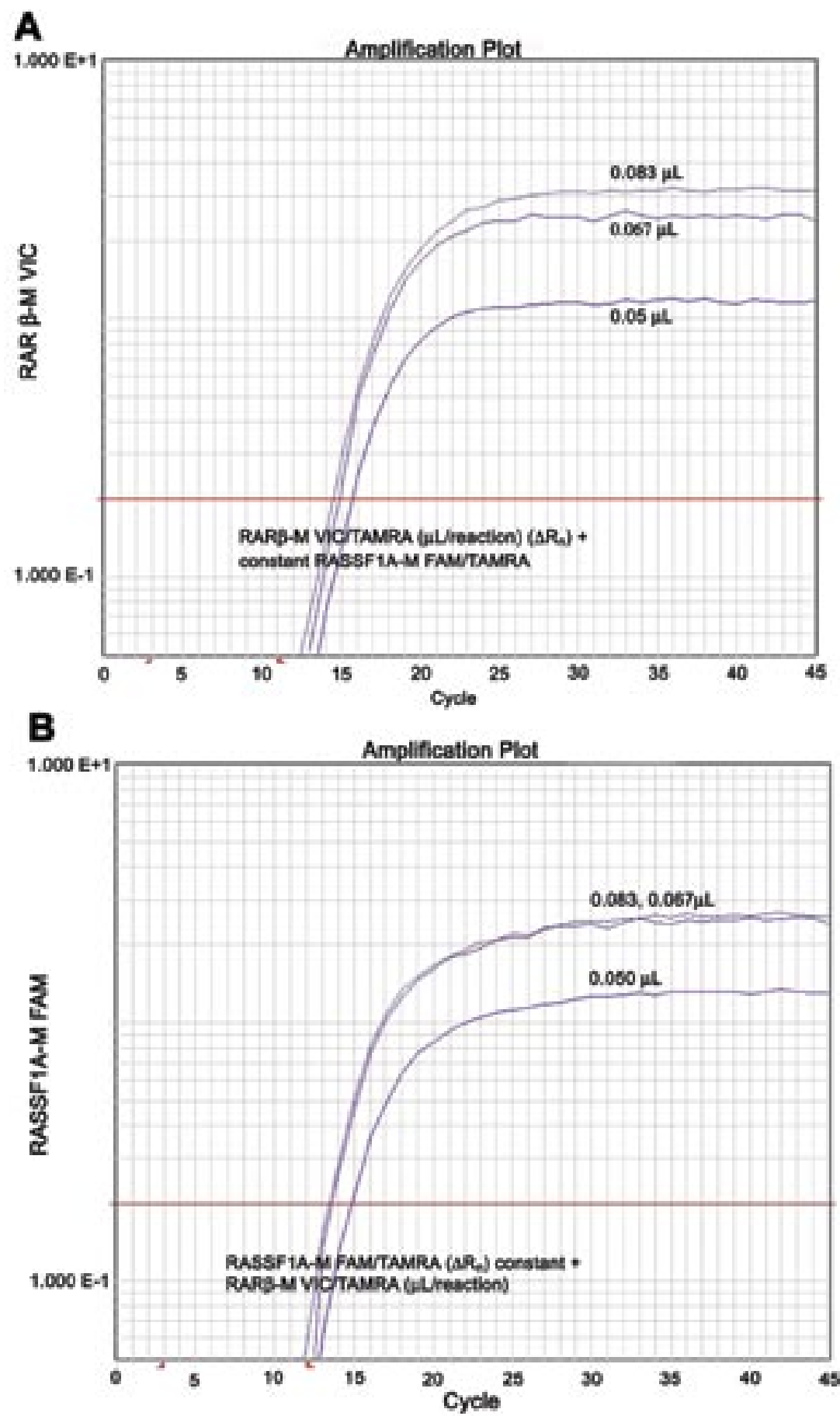

Figure 3. Optimization of two-color quantitative multiplex methylation-specific PCR (QM-MSP): varying concentrations of VIC/TAMRA-labeled probe affects both FAM and VIC signal intensity. To optimize two-color QM-MSP, the concentration of FAM/TAMRA-labeled probe was held constant at $0.05 \mu \mathrm{L} /$ reaction $(200 \mathrm{nM})$ while increasing concentrations $(0.05 \mu \mathrm{L}, 0.067 \mu \mathrm{L}, 0.083 \mu \mathrm{L} ; 200 \mathrm{nM}, 268$ $\mathrm{nM}$, and $332 \mathrm{nM}$, respectively) of VIC/TAMRA-labeled probe were tested. Shown are two amplification plots of the same well depicting the fluorescence intensity $\left(\Delta R_{n}\right)$ of RAR $\beta$-M VIC/TAMRA (A) and RASSF1A-M FAM/TAMRA (B). Plotted is $\Delta R_{n}$ versus PCR cycle. $y$-axis: $\Delta R_{n}$, fluorescence emission signal of FAM or VIC reporter dye/passive reference ROX dye across each real-time cycle; $\mathrm{x}$-axis: cycle, cycle number of the real-time PCR. The horizontal line indicates the threshold; cycle threshold $\left(\mathrm{C}_{\mathrm{T}}\right)$, cycle number at which the $R_{n}$ exceeds the baseline by the value of the threshold. comparable between singleplex and all three two-color real-time QM-MSP experiments (Table 1). The correlations between all three combinations as compared with one-color singleplex QM-MSP experiments for both the breast cancer and mammoplasty control samples were very high (range: 0.82 to 0.93 ), with $P$ values $<0.00001$ for each comparison. While percent DNA methylation values for all three permutations of the two-color method provided statistically similar data, we found our overall strongest qualitative and quantitative data came from using the $\mathrm{U}_{1}+\mathrm{M}_{1}$ primer/probe combination for the same gene (Table 1, Protocol C). For example, real-time co-amplification plots for the $\mathrm{U}_{1}+\mathrm{M}_{1}$ reaction were consistently the most robust in terms of signal strength (data not shown), and percent methylation values between one-color and twocolor QM-MSP were reproducible across the four genes analyzed (Table 1).

\section{DISCUSSION}

In this paper we demonstrate successful co-amplification of two methylation-specific target DNAs by utilizing FAM- and VIC-labeled probe sets in the same well without appreciable changes in percent methylation when compared with the singleplex real-time QM-MSP. To our knowledge, this is the first report for real-time MSP applications that either two genes or U+M primer/probe sets for the same gene have been simultaneously quantified in a single well. This modification can be applied to any real-time MSP and significantly decreases the cost and time of each experiment.

Our first undertaking was to identify compatible fluorophore-labeled probes that could be used to successfully coamplify two methylation-specific DNA targets in real-time. Unlike standard RT-PCR, we found that the unique challenges of the MSP had strong implications for co-amplification in real-time. Namely, the methylationspecific reaction is complicated by the generation of a relatively fragile singlestranded DNA template after $\mathrm{NaBi}$ treatment and the need to characterize and quantify similar U+M DNA targets 
for the same gene. The additional challenge presented in the real-time reaction is the requirement of $\mathrm{U}+\mathrm{M}$ real-time primers and probes with complementary $\mathrm{T}_{\mathrm{m}} \mathrm{s}$, while simultaneously limiting the competition for these similar DNA targets. Moreover, the number of spectrally resolved fluorophores that can maintain their spectral intensities during co-amplification is limited. For example, a number of reporter dye combinations such as JOE and VIC (Applied Biosystems) cannot be co-amplified in real-time due to either inferior spectral intensities of one probe partner or because the single excitation lasers used on the ABI PRISM 7700 and the 7900 platforms are unable to efficiently excite a wide range of fluorophores (22). In general, recommendations for successful co-amplification in real-time that apply to the three $\mathrm{A}, \mathrm{B}$, and $\mathrm{C}$ protocols described in this paper are to adjust primer concentrations, limit probe concentration of the more robust performing reporter dye, and most importantly, to choose probes with strong individual spectral intensities with nonoverlapping absorption spectra.

The rationale for testing all three $\mathrm{A}$, $\mathrm{B}$, and $\mathrm{C}$ protocols was 2 -fold. First, co-amplifying $\mathrm{U}_{1}+\mathrm{M}_{1}$ primers of the same gene had never been reported for any MSP application. Therefore if successful, the novelty of the $\mathrm{C}$ alternative would significantly reduce the costs and time involved in any real-time MSP application, especially for those researchers who only perform methylation studies on one gene. Second, for methylation studies that use a reference gene plus the target gene of interest (e.g., MethyLight), the $\mathrm{U}_{1}$ target could be replaced by a second reference gene such as actin. Given our preliminary data showing some decreased robustness with $U_{1}+U_{2}$ reactions, we

Table 1. Comparison of Percent Methylation Values for One- Versus Two-Color QM-MSP

\begin{tabular}{|c|c|c|c|c|c|c|c|c|}
\hline & \multicolumn{2}{|c|}{ TWIST } & \multicolumn{2}{|c|}{ Cyclin D2 } & \multicolumn{2}{|c|}{$R A R \beta$} & \multicolumn{2}{|c|}{ RASSF1A } \\
\hline & $\begin{array}{l}\text { One- } \\
\text { Color }\end{array}$ & $\begin{array}{l}\text { Two- } \\
\text { Colora }\end{array}$ & $\begin{array}{l}\text { One- } \\
\text { Color }\end{array}$ & $\begin{array}{l}\text { Two- } \\
\text { Colora }^{a}\end{array}$ & $\begin{array}{l}\text { One- } \\
\text { Color }\end{array}$ & $\begin{array}{l}\text { Two- } \\
\text { Colorb }\end{array}$ & $\begin{array}{l}\text { One- } \\
\text { Color }\end{array}$ & $\begin{array}{l}\text { Two- } \\
\text { Color }\end{array}$ \\
\hline \multicolumn{9}{|c|}{$\begin{array}{l}\text { Protocol A: } \mathrm{M}_{1}+\mathrm{M}_{2} \text { and } \mathrm{U}_{1}+\mathrm{U}_{2} \\
\text { (Two-Gene Reaction) }\end{array}$} \\
\hline Normal-1 & 0 & 0 & 0 & 0 & 0.4 & 0.6 & 0.6 & 0 \\
\hline Normal-2 & 0 & 0 & 0 & 0 & 1.7 & 1.3 & 4 & 3.6 \\
\hline Normal-3 & 0 & 0.1 & 0.9 & 3.4 & 0.1 & 0.2 & 1.6 & 1.4 \\
\hline Normal-4 & 0 & 0 & 0 & 0 & 0.6 & 0.6 & 0.1 & 0.1 \\
\hline HSD & 0 & 0 & 0 & 0 & 0 & 0 & 0 & 0 \\
\hline 231 & 100 & 100 & 100 & 100 & 100 & 100 & 100 & 100 \\
\hline Tumor-5 & 0 & 0 & 0 & 0 & 0 & 0 & 27 & 20 \\
\hline Tumor-6 & 31 & 28 & 1 & 0 & 22 & 26 & 9 & 4 \\
\hline Tumor-7 & 43 & 37 & 26 & 19 & 31 & 29 & 30 & 30 \\
\hline Tumor-8 & 9 & 10 & 0 & 0 & 21 & 39 & 31 & 15 \\
\hline HSD & 0 & 0 & 0 & 0 & 0 & 0 & 0 & 0 \\
\hline 231 & 100 & 100 & 100 & 100 & 100 & 100 & 100 & 100 \\
\hline \multicolumn{9}{|c|}{$\begin{array}{l}\text { Protocol B: } \mathrm{U}_{1}+\mathrm{M}_{2} \text { and } \mathrm{M}_{1}+\mathrm{U}_{2} \\
\text { (Two-Gene Reaction) }\end{array}$} \\
\hline Normal-1 & 0 & 0 & 0 & 0 & 0.4 & 0.4 & 0.6 & 0 \\
\hline Normal-2 & 0 & 0.1 & 0 & 0 & 1.7 & 0.7 & 4 & 4.1 \\
\hline Normal-3 & 0 & 0 & 0.9 & 1.7 & 0.1 & 0 & 1.6 & 0.2 \\
\hline Normal-4 & 0 & 0 & 0 & 0 & 0.6 & 0.5 & 0.1 & 0 \\
\hline HSD & 0 & 0 & 0 & 0 & 0 & 0 & 0 & 0 \\
\hline 231 & 100 & 100 & 100 & 100 & 100 & 100 & 100 & 100 \\
\hline Tumor-5 & 0 & 0 & 0 & 0 & 0 & 0 & 27 & 18 \\
\hline Tumor-6 & 31 & 31 & 1 & 0 & 22 & 23 & 9 & 4 \\
\hline Tumor-7 & 43 & 54 & 26 & 25 & 31 & 49 & 30 & 22 \\
\hline Tumor-8 & 9 & 8 & 0 & 0 & 21 & 27 & 31 & 23 \\
\hline HSD & 0 & 0 & 0 & 0 & 0 & 0 & 0 & 0 \\
\hline \multirow[t]{2}{*}{231} & 100 & 100 & 100 & 100 & 100 & 100 & 100 & 100 \\
\hline & & & & & \multicolumn{4}{|c|}{ Table 1. continued on next page } \\
\hline
\end{tabular}


Table 1. Continued from previous page

\begin{tabular}{|c|c|c|c|c|c|c|c|c|}
\hline & \multicolumn{2}{|c|}{ TWIST } & \multicolumn{2}{|c|}{ Cyclin D2 } & \multicolumn{2}{|c|}{$R A R \beta$} & \multicolumn{2}{|c|}{ RASSF1A } \\
\hline & $\begin{array}{l}\text { One- } \\
\text { Color }\end{array}$ & $\begin{array}{l}\text { Two- } \\
\text { Colora } \\
\end{array}$ & $\begin{array}{l}\text { One- } \\
\text { Color }\end{array}$ & $\begin{array}{l}\text { Two- } \\
\text { Colora } \\
\end{array}$ & $\begin{array}{l}\text { One- } \\
\text { Color }\end{array}$ & $\begin{array}{l}\text { Two- } \\
\text { Colorb }\end{array}$ & $\begin{array}{l}\text { One- } \\
\text { Color }\end{array}$ & $\begin{array}{l}\text { Two- } \\
\text { Color }\end{array}$ \\
\hline \multicolumn{9}{|c|}{$\begin{array}{l}\text { Protocol C: } \mathrm{U}_{1}+\mathrm{M}_{1} \\
\text { (One-Gene Reaction) }\end{array}$} \\
\hline Normal-1 & 0 & 0 & 0 & 0 & 0.4 & 1.5 & 0.6 & 1 \\
\hline Normal-2 & 0 & 0 & 0 & 0 & 1.7 & 0.9 & 4 & 6.3 \\
\hline Normal-3 & 0 & 0 & 0.9 & 0.6 & 0.1 & 0.4 & 1.6 & 0.5 \\
\hline Normal-4 & 0 & 0 & 0 & 0 & 0.6 & 0.2 & 0.1 & 0 \\
\hline HSD & 0 & 0 & 0 & 0 & 0 & 0 & 0 & 0 \\
\hline 231 & 100 & 100 & 100 & 100 & 100 & 100 & 100 & 100 \\
\hline Tumor-5 & 0 & 0 & 0 & 0 & 0 & 2 & 27 & 20 \\
\hline Tumor-6 & 31 & 32 & 1 & 0 & 22 & 30 & 9 & 16 \\
\hline Tumor-7 & 43 & 43 & 26 & 21 & 31 & 26 & 30 & 40 \\
\hline Tumor-8 & 9 & 12 & 0 & 0 & 21 & 19 & 31 & 21 \\
\hline HSD & 0 & 0 & 0 & 0 & 0 & 0 & 0 & 0 \\
\hline 231 & 100 & 100 & 100 & 100 & 100 & 100 & 100 & 100 \\
\hline \multicolumn{9}{|c|}{ 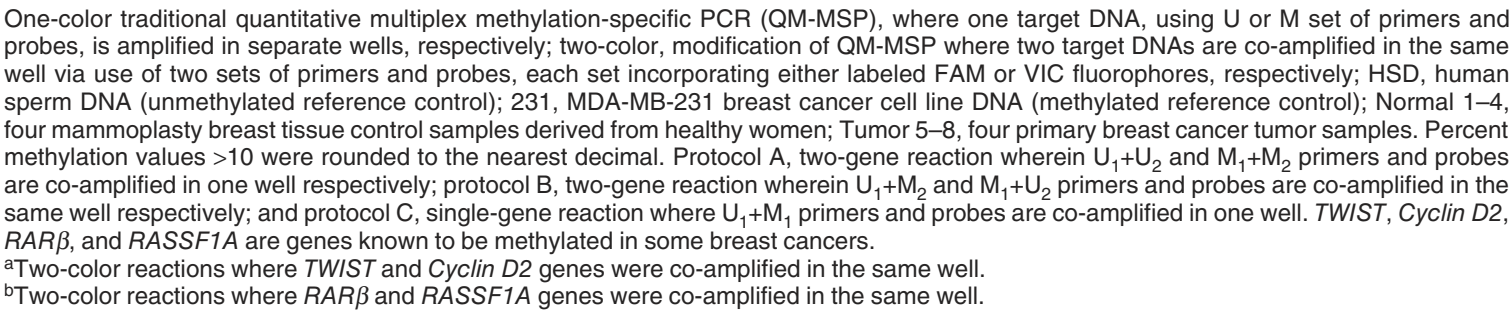 } \\
\hline
\end{tabular}

also thought it prudent to test both twogene A and B permutations thoroughly for any interactive effects.

In analyzing the $\mathrm{A}$ and $\mathrm{B}$ twogene experiments, we found on rare occasions that the $\mathrm{U}_{1}+\mathrm{U}_{2}$ two-gene reactions were somewhat less robust than the $\mathrm{M}_{1}+\mathrm{M}_{2}$ and $\mathrm{U}_{1}+\mathrm{M}_{2}$ two-gene reactions (data not shown). In one experiment, we obtained an erroneously inflated $\% \mathrm{M}$ for some two gene combinations based in part on weak $\mathrm{U}_{1}+\mathrm{U}_{2}$ reactions that rendered incorrectly low $\mathrm{U}$ values for a particular gene. Although the stringent use of unmethylated and methylated controls run on each real-time plate easily detected this discrepancy, such rare weak $\mathrm{U}_{1}+\mathrm{U}_{2}$ reactions, whether caused by interference, competition, or structural blocking, might best be overcome by better primer design.

Although specificity is a consideration in any multiplex reaction, we were especially concerned from a theoretical standpoint about the potential for cross-reactivity in the $\mathrm{U}_{1}+\mathrm{M}_{1}$ same-gene reaction through competition for 3 to $4 \mathrm{CpG}$ dinucleotide pairs typically covered by the average 27-bp real-time probe. We tested for this possibility and found that primer/ probe cross-reactivity did not occur. Notably, specificity for all three A, B, and $\mathrm{C}$ protocols was assured by several factors; the two-channel ABI PRISM Sequence Detection System (SDS) software (Applied Biosystems), the slopes of the standard curves generated with each two-color QM-MSP experiment, and percent methylation values obtained from methylated and unmethylated DNA controls. Because FAM and VIC reporter dyes each have their own detection channel, and only those fluorescent signals detectable within the range of the six-log standard curve were considered, we were able to overcome any potential concern with cross-reactivity between primer/probe sets for two DNA targets. In sum, these findings underscore of the impor- tance of choosing labeled probes with nonoverlapping absorption spectra, optimizing each gene reaction, incorporating appropriate controls on each real-time plate, and taking advantage of the dual channel detection intrinsic in the SDS real-time software.

QM-MSP allows quantification of DNA methylation for a panel of genes under conditions where DNA concentration is limited. However, this method is expensive in part because two sets of standard curves and controls occupy 36 of 96 wells on a real-time plate; a requirement when $U$ and $M$ reactions are performed in separate wells with one-color QM-MSP. Our modification of combining two real-time probes labeled with FAM and VIC reporter dyes can simultaneously quantify DNA methylation for either A, B, or $\mathrm{C}$ protocols and achieves a marked increase in efficiency and reduction in costs, without compromising specificity. In essence, progressing from a one-color to a two-color reaction allows 
for a doubling of either the number of genes that can be analyzed per sample for the A and B two-gene protocols or an increase in the number of samples analyzed per plate by a factor of 2.6 for the $\mathrm{C}$ same-gene protocol. For example, the cost for a one-color, onegene reaction for the second real-time step of QM-MSP (including the ABI 7900 machine fee, primer/probe sets, TaqMan polymerase, buffer reagents, 96-well reaction plate, and other laboratory supplies) is approximately $\$ 139.00$ per real-time plate. This cost is essentially cut in half with A and B protocols to $\$ 69.50 /$ plate, because two genes are analyzed simultaneously for each sample. For the A and B protocols, two standard curves plus controls are required that occupy 36 of 96 wells. The reason the cost for the $\mathrm{C}$ protocol is further reduced by a factor of 2.6 to approximately $\$ 53.00 /$ plate is that only one standard curve with controls (occupying only 18 of 96 wells) is generated because $\mathrm{U}_{1}+\mathrm{M}_{1}$ DNA targets for the same gene are co-amplified in the same well.

Future studies based on our modification would logically try to expand the two-color reaction to a three- or morecolor reaction. However, given the technical challenges of primer/probe optimization, competition for $\mathrm{U}+\mathrm{M}$ DNA targets, and the limitations of matching available TaqMan hydrolysis probes with appropriately different spectral resolutions and sufficient fluorescent intensities, it is likely that even a successful three-color reaction would be difficult to achieve for realtime MSP applications. Based on the low efficiency of co-amplification with CalO-labeled probes and the ABI data showing the inefficiency of JOE in a two-color reaction, we specifically recommend using FAM and VIC reporter dyes to carry out co-amplication for real-time MSP. Another future possibility would be to use a hybridization-based instead of a hydrolysis-based real-time chemistry in order to increase the number of different fluorophores that could successfully be co-amplified. In real-time hybridization reactions, primers rather than probes are labeled with donor and acceptor fluorophores to allow analysis of multiple $(>2)$ targets within the same reaction well (23) and therefore hold future promise for successfully being adapted to a number of quantitative MSP applications.

In conclusion, two methylationspecific DNA targets from either two genes, or $\mathrm{U}$ and $\mathrm{M}$ primers of the same gene, can be successfully co-amplified in the same well with FAM- and VIClabeled probes to accurately quantify methylation by QM-MSP. Because statistically similar percent methylation values are obtained with two-color $\mathrm{A}$, $\mathrm{B}$, and $\mathrm{C}$ reactions as with one-color singleplex reactions, any of these permutations of our improved method can be used. Moreover, the successful co-amplification of two DNA targets in the two-color QM-MSP method significantly saves time, resources, and costs. This technique may be used for candidate genes in a number of cancer types and is especially useful for quantification of methylation in DNA extracted from finite or scarce sources of tissues or cellular materials such as ductal lavage, nipple aspirates, archival histopathology slides, and cells obtained from laser capture microdissection (LCM). The two-color modification is applicable not only to QM-MSP, but to any MSP experiment performed in real-time. Any future application and translation of methylation analysis to the clinical setting will necessitate a reliable cost-effective method for quantifying methylation in human samples.

\section{ACKNOWLEDGMENTS}

This work was supported by National Institutes of Health/ National Cancer Insitute (NIH/NCI) Specialized Program of Research Excellence (SPORE) in Breast Cancer P50CA88843 to S.S. and NIH/National Institute of Nursing Research (NINR) F31 NR008311-01A1 and American Cancer Society (ACS) DSCN-04-16201 to T.S-S.

\section{COMPETING INTERESTS STATEMENT}

S.S. and M.J.F. are paid consultants for Oncomethylome Sciences. T.S-S. is listed as co-inventor on a patent ap- plication for which Oncomethylome Sciences holds an option to license. The terms of these arrangements are being managed with John Hopkins University in accordance with its conflict of interests policy. The other authors declare no competing interests.

\section{REFERENCES}

1. Ballestar, E. and M. Esteller. 2005. MethylCpG-binding proteins in cancer: blaming the DNA methylation messenger. Biochem. Cell Biol. 83:374-384.

2. Egger, G., G. Liang, A. Aparicio, and P.A. Jones. 2004. Epigenetics in human disease and prospects for epigenetic therapy. Nature 429:457-463.

3. Esteller, M. 2003. Cancer epigenetics: DNA methylation and chromatin alterations in human cancer. Adv. Exp. Med. Biol. 532:39-49.

4. Jones, P.A. 2003. Epigenetics in carcinogenesis and cancer prevention. Ann. NY Acad. Sci. 983:213-219.

5. Jones, P.A. and S.B. Baylin. 2002. The fundamental role of epigenetic events in cancer. Nat. Rev. Genet. 3:415-428.

6. Widschwendter, M. and P.A. Jones. 2002. DNA methylation and breast carcinogenesis. Oncogene 21:5462-5482.

7. Barcellos-Hoff, M.H. 2001. It takes a tissue to make a tumor: epigenetics, cancer and the microenvironment. J. Mammary Gland Biol. Neoplasia 6:213-221.

8.Jones, P.A. and P.W. Laird. 1999. Cancer epigenetics comes of age. Nat. Genet. 21:163-167.

9. Thomassin, H., C. Kress, and T. Grange. 2004. MethylQuant: a sensitive method for quantifying methylation of specific cytosines within the genome. Nucleic Acids Res. 32:e168.

10. Frommer, M., L.E. McDonald, D.S. Millar, C.M. Collis, F. Watt, G.W. Grigg, P.L. Molloy, and C.L. Paul. 1992. A genomic sequencing protocol that yields a positive display of 5-methylcytosine residues in individual DNA strands. Proc. Natl. Acad. Sci. USA 89:1827-1831.

11. Clark, S.J., J. Harrison, C.L. Paul, and M. Frommer. 1994. High sensitivity mapping of methylated cytosines. Nucleic Acids Res. 22:2990-2997.

12. Herman, J.G., J.R. Graff, S. Myohanen, B.D. Nelkin, and S.B. Baylin. 1996 Methylation-specific PCR: a novel PCR assay for methylation status of $\mathrm{CpG}$ islands. Proc. Natl. Acad. Sci. USA 93:9821-9826.

13. Ahuja, N., Q. Li, A.L. Mohan, S.B. Baylin, and J.P. Issa. 1998. Aging and DNA methylation in colorectal mucosa and cancer. Cancer Res. 58:5489-5494.

14. Lehmann, U., F. Langer, H. Feist, S. Glockner, B. Hasemeier, and H. Kreipe. 2002. Quantitative assessment of promoter hypermethylation during breast cancer development. Am. J. Pathol. 160:605-612.

15. Fackler, M.J., M. McVeigh, J. Mehrotra, M.A. Blum, J. Lange, A. Lapides, E. Garrett, P. Argani, and S. Sukumar. 2004. Quantitative multiplex methylation-specific 
PCR assay for the detection of promoter hypermethylation in multiple genes in breast cancer. Cancer Research. 64:4442-4452.

16.Eads, C.A., K.D. Danenberg, K. Kawakami, L.B. Saltz, C. Blake, D. Shibata, P.V. Danenberg, and P.W. Laird. 2000. MethyLight: a high-throughput assay to measure DNA methylation. Nucleic Acids Res. 28:e32.

17. Cottrell, S.E., J. Distler, N.S. Goodman, S.H. Mooney, A. Kluth, A. Olek, I. Schwope, R. Tetzner, et al. 2004. A realtime PCR assay for DNA-methylation using methylation-specific blockers. Nucleic Acids Res. 32:e10.

18. Thomassin, H., C. Kress, and T. Grange. 2004. MethylQuant: a sensitive method for quantifying methylation of specific cytosines within the genome. Nucleic Acids Res. 32:e168.

19. Hindiyeh, M., V. Levy, R. Azar, N. Varsano, L. Regev, Y. Shalev, Z. Grossman, and E. Mendelson. 2005. Evaluation of a multiplex real-time reverse transcriptase PCR assay for detection and differentiation of influenza viruses A and B during the 2001-2002 influenza season in Israel. J. Clin. Microbiol. 43:589-595.

20. Simi, L., M. Andreani, F. Davini, A. Janni, M. Pazzagli, M. Serio, and C. Orlando. 2004. Simultaneous measurement of MMP9 and TIMP1 mRNA in human non small cell lung cancers by multiplex real time RT-PCR. Lung Cancer 45:171-179.

21. Grace, M.B., C.B. McLeland, S.J. Gagliardi, J.M. Smith, W.E. Jackson III, and W.F. Blakely. 2003. Development and assessment of a quantitative reverse transcription-PCR assay for simultaneous measurement of four amplicons. Clin. Chem. 49:1467-1475

22. Bustin, S.A. 2002. Quantification of mRNA using real-time reverse transcription PCR (RT-PCR): trends and problems. J. Mol. Endocrinol. 29:23-39.

23. Wong, M.L. and J.F. Madrano. 2005. Real-time PCR for mRNA quantitation. BioTechniques 39:75-85.

24.Zeger, S.L. and K.Y. Liang. 1986 Longitudinal data analysis for discrete and continuous outcomes. Biometrics 42:121-130.

Received 15 August 2005; accepted 7 November 2005.

Address correspondence to:

Mary Jo Fackler

Sidney Kimmel Comprehensive Cancer Center at Johns Hopkins

CRB Room 416

1650 Orleans Street

Baltimore, MD 21231-1000, USA

e-mail:mfackler@jhmi.edu

To purchase reprints

of this article, contact

Reprints@BioTechniques.com 